\section{Asia's tigers get the blues}

Mental illness, in

particular depression, seems to be on the rise throughout East Asia. Why is this so? And can psychiatrists and the pharmaceutical industry together turn back the tide? Carina Dennis investigates.

n China, old people are killing themselves at an unprecedented rate. Once venerable and respected, the elderly have been left feeling uncertain of their status as rapid economic development has transformed family and community structures. Many give up hope, and end their lives with a dose of agricultural pesticide. "The suicide rate among the elderly in parts of Asia is shocking," says Helen Chiu, a psychiatrist at the Chinese University of Hong Kong.

In Japan, meanwhile, roughly a million youths have turned their backs on society. Some lock themselves in their rooms to sleep all day and play computer games all night. This social phobia — known as hikikomori - is seen as a symptom of a young generation unable to cope with socio-economic uncertainties. The decades-long Japanese economic boom has collapsed, with the emergence of an unemployed underclass.

These distressing phenomena are just part of what seems to be a general upward swing in the rates of mental illness being recorded across East Asia. In part, the explanation lies with rapid societal changes that have swept through the region as many of its nations have flexed their economic muscle. But some experts argue that recent studies may also have unmasked long-standing issues with mental health that went unrecognized in societies that have traditionally tended to view psychiatric illness as a sign of moral weakness.

Whatever the explanation, many Asian nations are currently ill-equipped to provide the specialist care needed to rehabilitate those who are suffering from mental illness. East Asian people also tend to possess genetic profiles that make them respond very differently to the antidepressant and antipsychotic

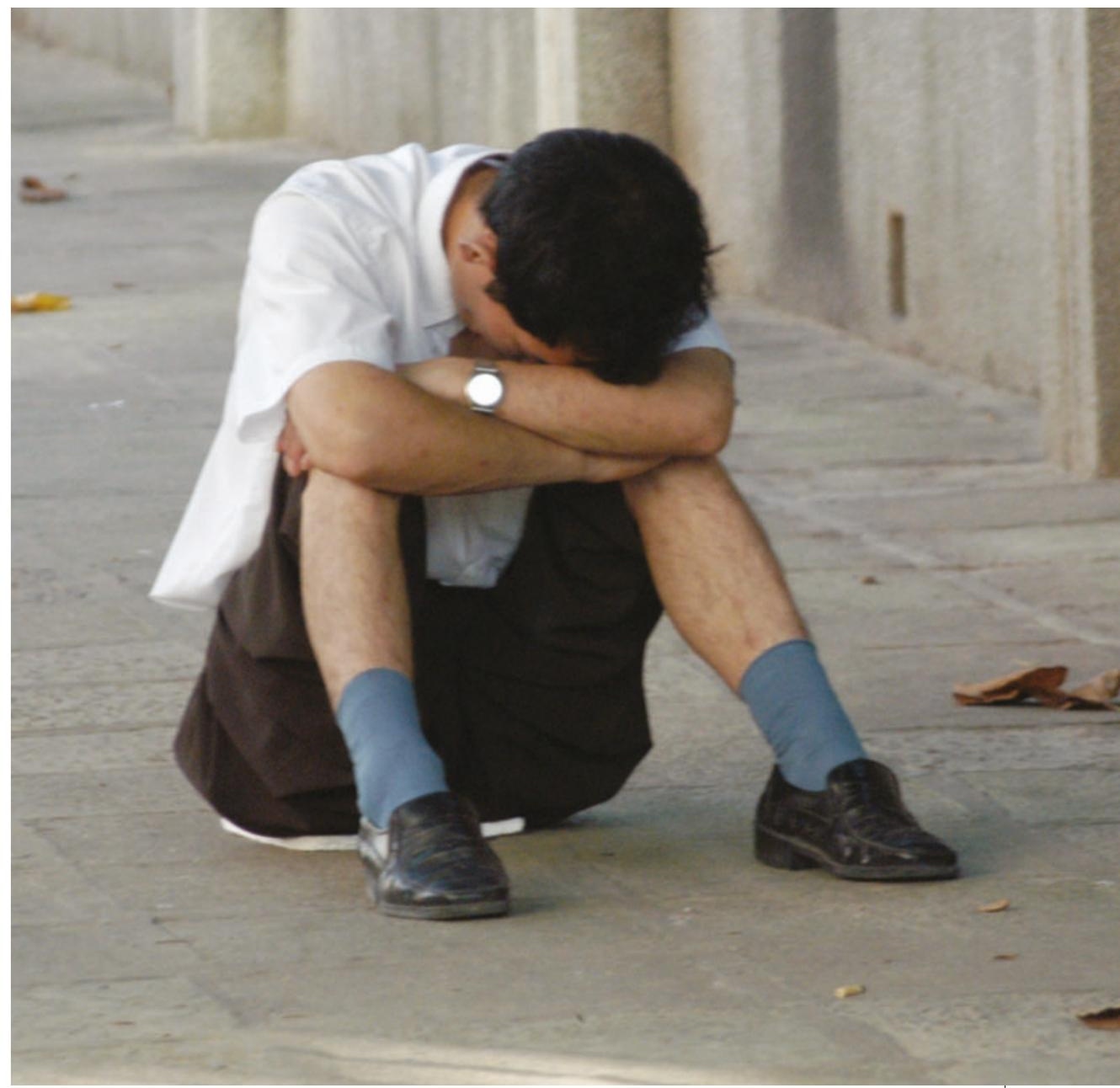

drugs developed to help bring relief to Westerners. If the region's problems with mental health are to be tackled, psychiatrists, geneticists and the pharmaceutical industry all have a lot of work to do.

Severe mental illnesses, such as schizophrenia and other psychotic disorders, are relatively easily recognized and are thought to occur at similar rates in societies throughout the world. But when psychiatrists first cast their gaze East in the 1950s, they concluded that Asia was almost entirely free of the subtler mood disorders that are prevalent in most Western societies ${ }^{1,2}$.

Even the most recent data gathered for the World Health Organization and published this month ${ }^{3}$ suggest that people in China and Japan suffer less from depression and related disorders than their Western counterparts. But some experts argue that these figures fail to reflect the true magnitude of the problem - particularly given that Asia has some of the highest suicide rates in the world (see Figure, opposite). "If we consider suicide as an extreme endpoint of depression and other mood disorders, it suggests that we cannot have less of a problem with mental illness than the West," says Sing Lee, a psychiatrist at the Chinese University of Hong Kong.

Nearly 300,000 Chinese kill themselves each year, mostly in rural areas ${ }^{4}$. It is one of the few countries in which more women than men commit suicide - accounting for more than half of all female suicides worldwide ${ }^{5}$. Michael Phillips, a psychiatrist at the Beijing Suicide Research and Prevention Center at Hui Long Guan Hospital, suspects that the high suicide rate in China is in part due to attempted suicides being more successful than in the West. "I don't believe all those people wanted to kill themselves," he says. Rather, he argues that the highly toxic pesticides widely used in rural China for suicide attempts and scant resuscitation facilities lead to high fatalities from what might elsewhere be considered a cry for help.

\section{Broken homes}

Chiu links the apparent rise in suicide with the changing socio-economic climate across East Asia. This has brought diminished financial and status security, rising unemployment and the breakdown of traditional family and community networks. Large, tightly knit family groups have been replaced by nuclear families that move from their communities in search of work, often great distances away. And in China, the breakdown of family-support networks may be set to escalate, as the one-child policy, introduced in 1979 to control population growth, makes its effects felt. 
anxiety disorders and other mental illnesses across Asia. But tailoring antidepressants developed for Western populations to Asian patients will require research into genetics.

"In general, most Asian patients respond to lower doses of psychotropic drugs than Caucasians," says Keh-Ming Lin, who heads the Division of Mental Health and Drug Abuse Research at the National Health Research Institutes in Taipei, Taiwan. In some instances, the dose can be halved.

Lin was among the first psychiatrists to recognize that drugs used to treat mental illness have different effects across ethnic boundaries. More than 15 years ago, while working at the University of California at Los Angeles, he found evidence that Asian patients metabolize haloperidol, commonly used to treat schizophrenia, more slowly than Caucasians'. Asian patients are also more likely to develop severe side effects when treated with antipsychotic drugs ${ }^{10}$.

Unpublished work from Chee $\mathrm{Ng}$, a psychiatrist at the University of Melbourne, suggests that it's a similar story for antidepressants. He has found that patients of Chinese origin respond to lower doses of an anti-depressant called sertraline. These patients were also more likely to drop out of the study. "They may experience more side effects at regular doses and therefore be less compliant in taking the medication," Ng suggests.

These phenomena are probably due to genetic differences that alter the activity of enzymes that metabolize drugs and allow them to be removed from the body. The most striking examples are the cytochrome P450 enzymes in the liver and, in particular, an enzyme called CYP2D6. "Almost half of

the drugs used in psychiatry are metabolized by CYP2D6," says Lin, adding that up to $70 \%$ of Asians harbour subtle mutations that impair the enzyme's activity.

Rather than making gross generalizations about the response of Asian patients to drugs, geneticists argue that more detailed studies are needed to look at the relationship between genetic make-up and drug response for each pharmaceutical in different populations. In these studies, it will be important to take account of environmental factors, including diet, smoking and alcohol consumption, which can affect an individual's response to a drug.

Another key variable is whether patients are supplementing their medication with herbal remedies, which can interfere with the metabolism of other drugs. In many Asian cultures, it is common for patients to try traditional medicine before turning to Western medicine. "We are not the first choice," says
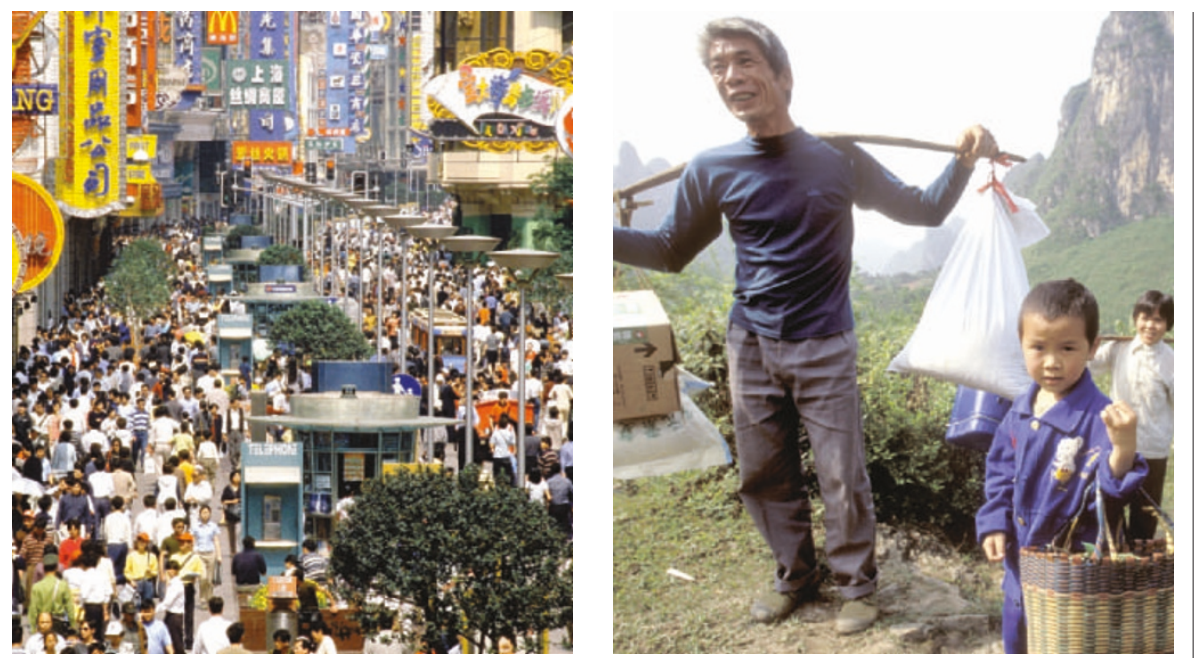

Worlds apart: different methods are needed to diagnose psychiatric illness in urban and rural China.

Nor Hayati Ali, a psychiatrist at Kuala Lumpur Hospital in Malaysia. "Most patients still consult a traditional healer first."

\section{Cultural revolution}

Understanding how different populations respond to different medicines is just one challenge for researchers tackling mental illness in Asia. Different cultures are likely to respond differently to other treatments, such as psychotherapy and behavioural therapies. So psychiatrists will need to investigate how to tailor these therapies to work effectively in various Asian countries.

Researchers also need to get better estimates of the prevalence of mental disorders across the region. Epidemiological studies have so far been hampered by inconsistencies in the diagnostic criteria used. Improving matters will require the devel-opment of diagnostic tools that are sensitive to different ways in which mental disorders can manifest themselves in different Asian cultures.

Although Asia's problems with mental illness are daunting, psychiatrists such as Phillips are inspired by the knowledge that they can make a difference. A Canadian national, Phillips first visited China in 1976 with friends when he was a recently qualified doctor working in New Zealand.

Months later, he returned to China on a fellowship with the goal of studying how government propaganda is used to promulgate public-health issues, gaining information he hoped to apply in another developing region. In the event, the Chinese authorities did not let this project go ahead, but Phillips stayed nonetheless and learnt Mandarin Chinese. Within two years he was hooked, both on Chinese culture and the feeling that he could make a difference.

After moving to the United States to train in psychiatry, epidemiology and anthropology, Phillips settled in Beijing in 1985. In the early days, he was regarded with mistrust. "There was a suspicion that I might be a spy," he says. But today, he finds that being a foreigner has some advantages, allowing him to be more outspoken in his relations with the media than his Chinese colleagues. "It helps me get my message out," he says.

Phillips' message is getting through: the Chinese authorities now support his research. In one project, he has interviewed a sample of 1,000 people from the general population to understand the factors that underlie the stigma associated with suicide. "To change attitudes, you need to know what the attitudes are," he says. In another study, his team is following up on rural people who have attempted suicide, and offering them social support, such as trying to ameliorate family crises that can underlie their mentalhealth problems. The goal is to develop a model for suicide prevention in rural China.

"Suicide is the number one cause of death in young Chinese, and up until ten years ago no one was doing anything about it," says Phillips. Now numerous hotlines and prevention programmes are starting to spring up. "China is changing so rapidly," he says. "It is amazing to watch and to be part of it." Carina Dennis is Nature's Australasian correspondent.

1. Lin, K-M. \& Cheung, F. Psychiatr. Serv. 50, 774-780 (1999).

2. Kleinman, A. Social Origins of Distress and Disease: Depression, Neurasthenia, and Pain in Modern China (Yale Univ. Press, New Haven, Connecticut, 1986).

3. The WHO World Mental Health Survey Consortium. J. Am Med. Assoc. 291, 2581-2590 (2004).

4. Phillips, M. R., Li, X. \& Zhang, Y. Lancet 359, 835-840 (2002).

5. Phillips, M. R., Liu, H. Q. \& Zhang, Y. P. Cult. Med. Psychiatr. 23, 25-50 (1999).

6. Kleinman, A. Cult. Med. Psychiatr. 6, 117-189 (1982).

7. Report on the WHO Collaborative Study on Standardized Assessment of Depressive Disorders (WHO, Geneva, 1983).

8. The World Health Report 2001 (WHO, Geneva, 2001)

9. Lin, K-M. et al. J. Clin. Psychopharmacol. 8, 195-201 (1988).

10. Lin, K.-M. \& Poland, R. E. in Psychopharmacology: The Fourth Generation of Progress (eds Bloom, F. E. \& Kupfer, D. J.) 1907-1917 (Raven, New York, 1995). 